Supporting Information for

\title{
Syntheses and synthetic applications of stannylated
}

\section{allylic alcohols}

\author{
Uli Kazmaier*, Simon Lucas, Manuela Klein
}

Institut für Organische Chemie, Universität des Saarlandes, D-66123 Saarbrücken, Germany.

E-mail: u.kazmaier@mx.uni-saarland.de 


\section{Table of contents}

Analytical and detailed NMR-data of compounds 13-17 $\quad$ S3

(E/Z)-1-Phenyl-4-tributylstannylbut-2-en-1-ol (13a) $\quad$ S3

(E/Z)-1-(4-Methylphenyl)-4-tributylstannylbut-2-en-1-ol (13b) S3

(E/Z)-1-(2-Methoxyphenyl)-4-tributylstannylbut-2-en-1-ol (13c) $\quad$ S4

(Z)-4-Tributylstannyl-1-(2,4,6-trimethoxy-phenyl)-but-2-en-1-ol (13d) S4

(E/Z)-1-(2-Bromophenyl)-4-tributylstannylbut-2-en-1-ol (13e) $\quad$ S4

(E/Z)-1-(2-Chloro-phenyl)-4-tributylstannyl-but-2-en-1-ol (13f) S5

(E/Z)-1-(2,6-Dichloro-phenyl)-4-tributylstannyl-but-2-en-1-ol (13g) S5

(E/Z)-1-(2-Nitro-phenyl)-4-tributylstannyl-but-2-en-1-ol (13h) S5

(E/Z)-1-(4-Nitrophenyl)-4-tributylstannylbut-2-en-1-ol (13i) S6

(E/Z)-1-Phenyl-5-tributylstannylpent-3-en-2-ol (13k) S6

(E/Z)-6-Methyl-1-tributylstannylhept-2-en-4-ol (13I) $\quad$ S7

(E/Z)-1-(Cyclohex-3-en-1-yl)-4-tributylstannylbut-2-en-1-ol (13m) S7

1-Phenylbuta-1,3-diene (14a) $\quad$ S8

3-Tributylstannanyl-1-(2,4,6-trimethoxy-phenyl)-but-3-en-1-ol (15d) S8

(E)-4-Iodo-1-phenylbut-2-en-1-ol (16a) $\quad$ S8

(E)-4-Iodo-1-(2-nitro-phenyl)-but-2-en-1-ol (16h) $\quad \mathrm{S} 8$

(E)-4-Iodo-1-(4-nitrophenyl)-but-2-en-1-ol (16i) $\quad$ S8

cis/trans-2-(4-Methylphenyl)-3-vinyloxiran (17b) S9

cis/trans-2-(2-Methoxyphenyl)-3-vinyloxiran (17c) S9

cis/trans-2-(2-Bromophenyl)-3-vinyloxiran (17e) S10

$\begin{array}{lr}\text { trans-2-(4-Nitrophenyl)-3-vinyloxiran (17i) } & \text { S10 }\end{array}$

$\begin{array}{lr}\text { trans-2-Phenylmethyl-3-vinyloxiran (17k) } & \text { S10 }\end{array}$ 


\section{General Experimental Methods}

All reactions were carried out in oven-dried glassware $\left(100^{\circ} \mathrm{C}\right)$ under argon. All solvents were dried before use. THF was distilled from $\mathrm{LiAlH}_{4}$ and stored over molecular sieves. The products were purified by flash chromatography on silica gel. Mixtures of EtOAc and hexanes were generally used as eluents, $1 \% \mathrm{NEt}_{3}$ was added for the purification of the stannanes. TLC: commercially precoated Polygram ${ }^{\circledR}$ SIL-G/UV 254 plates. Visualization was accomplished with UV-light and $\mathrm{KMnO}_{4}$ solution. Melting points are uncorrected. Selected signals in the NMR spectra for the minor isomers are extracted from the spectra of the isomeric mixture. Elemental analyses were carried out at the department of chemistry, University of Saarbrücken.

\section{Analytical and detailed NMR-data of compounds 13-17}

(E/Z)-1-Phenyl-4-tributylstannylbut-2-en-1-ol (13a): According to the general procedure for hydrostannations 13a was obtained from allenol 12a (198 mg, $1.50 \mathrm{mmol})$ as a colorless liquid (463 $\mathrm{mg}, 1.06 \mathrm{mmol}, 71 \%)$ and a 2:3 (E/Z)-mixture. (Z)-13a: ${ }^{1} \mathrm{H}$ NMR (500 MHz, $\left.\mathrm{CDCl}_{3}\right): \delta=0.82-0.89$ (m, 15H, 1-H, 4-H), 1.22-1.30 (m, 6H, 3-H), 1.41-1.47 (m, 6H, 2-H), 1.74 (m, 2H, 5-H), 1.77 (d, $\left.{ }^{3} J_{\mathrm{OH}, 8}=3.5 \mathrm{~Hz}, 1 \mathrm{H}, \mathrm{OH}\right), 5.37(\mathrm{~m}, 1 \mathrm{H}, 7-\mathrm{H}), 5.50\left(\mathrm{dd},{ }^{3} J_{8,7}=8.5 \mathrm{~Hz},{ }^{3} J_{8, \mathrm{OH}}=3.5 \mathrm{~Hz}, 1 \mathrm{H}, 8-\mathrm{H}\right), 5.75$ $(\mathrm{m}, 1 \mathrm{H}, 6-\mathrm{H}), 7.22-7.40(\mathrm{~m}, 5 \mathrm{H}, 10-\mathrm{H}, 11-\mathrm{H}, 12-\mathrm{H}) ;{ }^{13} \mathrm{C} \mathrm{NMR}\left(125 \mathrm{MHz}, \mathrm{CDCl}_{3}\right): \delta=9.4(\mathrm{t}, \mathrm{C}-1)$, 11.3 (t, C-5), 13.7 (q, C-4), 27.3 (t, C-3), 29.1 (t, C-2), 69.5 (d, C-8), 126.1 (d, C-6), 126.4 (d, C-10), 127.8 (d, C-12), 128.4 (d, C-11), 132.1 (d, C-7), 144.2 (s, C-9); (E)-13a (selected signals): ${ }^{1} \mathrm{H}$ NMR $\left(500 \mathrm{MHz}, \mathrm{CDCl}_{3}\right): \delta=5.12\left(\mathrm{dd},{ }^{3} J_{8,7}=7.4 \mathrm{~Hz},{ }^{3} J_{8, \mathrm{OH}}=3.5 \mathrm{~Hz}, 1 \mathrm{H}, 8-\mathrm{H}\right), 5.46\left(\mathrm{dd},{ }^{3} J_{7,6}=15.0 \mathrm{~Hz}\right.$, $\left.{ }^{3} J_{7,8}=7.4 \mathrm{~Hz},{ }^{4} J_{7, \mathrm{Sn}}=37.5 \mathrm{~Hz}, 1 \mathrm{H}, 7-\mathrm{H}\right), 5.85\left(\mathrm{dt},{ }^{3} J_{6,7}=15.0 \mathrm{~Hz},{ }^{3} J_{6,5}=8.8 \mathrm{~Hz},{ }^{3} J_{6, \mathrm{Sn}}=49.8 \mathrm{~Hz}, 1 \mathrm{H}\right.$, 6-H); ${ }^{13} \mathrm{C}$ NMR (125 MHz, $\mathrm{CDCl}_{3}$ ): $\delta=9.3$ (t, C-1), 13.6 (q, C-4), 14.5 (t, C-5), 29.0 (t, C-2), 75.6 (d, C-8), 126.0 (d, C-6), 128.3 (d, C-11), 133.0 (d, C-7), 144.0 (s, C-9); HRMS (CI): calcd. for $\mathrm{C}_{18} \mathrm{H}_{29} \mathrm{O}^{120} \mathrm{Sn}[\mathrm{M}-\mathrm{Bu}]^{+}$381.1186; found 381.1213 .

(E/Z)-1-(4-Methylphenyl)-4-tributylstannylbut-2-en-1-ol (13b): According to the general procedure for hydrostannations $\mathbf{1 3 b}$ was obtained from allenol $\mathbf{1 2 b}(50 \mathrm{mg}, 0.50 \mathrm{mmol})$ as a colorless liquid (176 mg, $0.39 \mathrm{mmol}, 78 \%)$ and a 1:1 (E/Z)-mixture. As a side product 14b was obtained in 5\% yield. $(E)-13 \mathrm{~b}:{ }^{1} \mathrm{H}$ NMR $\left(500 \mathrm{MHz}, \mathrm{CDCl}_{3}\right): \delta=0.81-0.88(\mathrm{~m}, 15 \mathrm{H}, 1-\mathrm{H}, 4-\mathrm{H}), 1.23-1.30(\mathrm{~m}$, 6H, 3-H), 1.40-1.49 (m, 6H, 2-H), $1.69\left(\mathrm{~d},{ }^{3} J_{\mathrm{OH}, 8}=3.5 \mathrm{~Hz}, 1 \mathrm{H}, \mathrm{OH}\right), 1.72-1.91(\mathrm{~m}, 2 \mathrm{H}, 5-\mathrm{H}), 2.32$ $(\mathrm{s}, 3 \mathrm{H}, 13-\mathrm{H}), 5.08\left(\mathrm{dd},{ }^{3} J_{8,7}=7.3 \mathrm{~Hz},{ }^{3} J_{8, \mathrm{OH}}=3.5 \mathrm{~Hz}, 1 \mathrm{H}, 8-\mathrm{H}\right), 5.46\left(\mathrm{dd},{ }^{3} J_{7,6}=15.8 \mathrm{~Hz},{ }^{3} J_{7,8}=7.3\right.$ $\mathrm{Hz}, 1 \mathrm{H}, 7-\mathrm{H}), 5.85\left(\mathrm{ddt},{ }^{3} J_{6,7}=15.8 \mathrm{~Hz},{ }^{3} J_{6,5}=8.5 \mathrm{~Hz},{ }^{4} J_{6,8}=0.6 \mathrm{~Hz},{ }^{3} J_{6, \mathrm{Sn}}=50.1 \mathrm{~Hz}, 1 \mathrm{H}, 6-\mathrm{H}\right)$, 7.11-7.28 (m, 4H, 10-H, 11-H); ${ }^{13} \mathrm{C}$ NMR (125 MHz, $\left.\mathrm{CDCl}_{3}\right): \delta=9.3$ (t, C-1), 13.7 (q, C-4), $14.4(\mathrm{t}$, C-5), 21.1 (q, C-13), 27.3 (t, C-3), 29.1 (t, C-2), 75.6 (d, C-8), 125.9 (d, C-10), 126.5 (d, C-6), 129.0 (d, C-11), 131.8 (d, C-7), 136.8 (s, C-12), 141.0 (s, C-9); (Z)-13b (selected signals): ${ }^{1} \mathrm{H}$ NMR (500 
$\left.\mathrm{MHz}, \mathrm{CDCl}_{3}\right): \delta=1.65\left(\mathrm{~d},{ }^{3} J_{\mathrm{OH}, 8}=3.5 \mathrm{~Hz}, 1 \mathrm{H}, \mathrm{OH}\right), 5.36(\mathrm{~m}, 1 \mathrm{H}, 7-\mathrm{H}), 5.46(\mathrm{~m}, 1 \mathrm{H}, 8-\mathrm{H}), 5.73(\mathrm{~m}$, $1 \mathrm{H}, 6-\mathrm{H}) ;{ }^{13} \mathrm{C}$ NMR $\left(125 \mathrm{MHz}, \mathrm{CDCl}_{3}\right): \delta=9.4(\mathrm{t}, \mathrm{C}-1), 11.3(\mathrm{t}, \mathrm{C}-5), 69.5$ (d, C-8), 126.1 (d, C-10), 127.9 (d, C-6), 129.1 (d, C-11), 132.7 (d, C-7), 137.0 (s, C-12), 141.3 (s, C-9); HRMS (CI): calcd. for $\mathrm{C}_{19} \mathrm{H}_{31} \mathrm{O}^{120} \mathrm{Sn}[\mathrm{M}-\mathrm{Bu}]^{+}$395.1371; found 395.1368.

(E/Z)-1-(2-Methoxyphenyl)-4-tributylstannylbut-2-en-1-ol (13c): According to the general procedure for hydrostannations $\mathbf{1 3 c}$ was obtained from allenol $\mathbf{1 2 c}(88 \mathrm{mg}, 0.50 \mathrm{mmol})$ as a colorless liquid (146 mg, $0.31 \mathrm{mmol}, 62 \%)$ and a 2:5:1 E/Z/diene-mixture. (Z)-13c: ${ }^{1} \mathrm{H}$ NMR (500 MHz, $\left.\mathrm{CDCl}_{3}\right): \delta=0.82-0.89(\mathrm{~m}, 15 \mathrm{H}, 1-\mathrm{H}, 4-\mathrm{H}), 1.23-1.31(\mathrm{~m}, 6 \mathrm{H}, 3-\mathrm{H}), 1.39-1.48(\mathrm{~m}, 6 \mathrm{H}, 2-\mathrm{H}), 1.80$ $(\mathrm{m}, 2 \mathrm{H}, 5-\mathrm{H}), 2.69\left(\mathrm{~d},{ }^{3} J_{\mathrm{OH}, 8}=5.4 \mathrm{~Hz}, 1 \mathrm{H}, \mathrm{OH}\right), 3.84(\mathrm{~s}, 3 \mathrm{H}, 15-\mathrm{H}), 5.48\left(\mathrm{dd},{ }^{3} J_{7,6}=10.4 \mathrm{~Hz},{ }^{3} J_{7,8}=\right.$ $8.5 \mathrm{~Hz}, 1 \mathrm{H}, 7-\mathrm{H}), 5.67\left(\mathrm{dd},{ }^{3} J_{8,7}=8.5 \mathrm{~Hz},{ }^{3} J_{8, \mathrm{OH}}=5.4 \mathrm{~Hz}, 1 \mathrm{H}, 8-\mathrm{H}\right), 5.74(\mathrm{~m}, 1 \mathrm{H}, 6-\mathrm{H}), 6.87$ (m, $13-$ $\mathrm{H}), 6.93(\mathrm{~m}, 11-\mathrm{H}), 7.23(\mathrm{~m}, 12-\mathrm{H}), 7.31(\mathrm{~m}, 10-\mathrm{H}) ;{ }^{13} \mathrm{C} \mathrm{NMR}\left(125 \mathrm{MHz}, \mathrm{CDCl}_{3}\right): \delta=9.3(\mathrm{t}, \mathrm{C}-1)$, 11.3 (t, C-5), 13.7 (q, C-4), 27.3 (t, C-3), 29.1 (t, C-2), 55.3 (q, C-15), 66.6 (d, C-8), 110.6 (d, C-13), 120.8 (d, C-11), 125.2 (d, C-10), 127.4 (d, C-6), 128.4 (d, C-12), 128.6 (s, C-9), 131.5 (d, C-7), 156.9 (s, C-14); (E)-13c (selected signals): $\left.{ }^{1} \mathrm{H} \mathrm{NMR} \mathrm{(500} \mathrm{MHz,} \mathrm{CDCl}_{3}\right): \delta=2.62\left(\mathrm{~d},{ }^{3} J_{\mathrm{OH}, 8}=6.0 \mathrm{~Hz}\right.$, $1 \mathrm{H}, \mathrm{OH}), 3.85(\mathrm{~s}, 3 \mathrm{H}, 15-\mathrm{H}), 5.30(\mathrm{~m}, 1 \mathrm{H}, 8-\mathrm{H}), 5.56\left(\mathrm{dd},{ }^{3} J_{7,6}=15.1 \mathrm{~Hz},{ }^{3} J_{7,8}=6.9 \mathrm{~Hz}, 1 \mathrm{H}, 7-\mathrm{H}\right)$, $5.80(\mathrm{~m}, 1 \mathrm{H}, 6-\mathrm{H}) ;{ }^{13} \mathrm{C} \mathrm{NMR}\left(125 \mathrm{MHz}, \mathrm{CDCl}_{3}\right): \delta=9.2$ (t, C-1), 14.4 (t, C-5), 29.0 (t, C-2), 55.4 (q, C-15), 72.1 (d, C-8), 110.5 (d, C-13), 120.7 (d, C-11), 127.2 (d, C-6), 128.2 (d, C-12), 130.2 (s, C-9), 132.0 (d, C-7), 156.5 (s, C-14); HRMS (CI): calcd. for $\mathrm{C}_{19} \mathrm{H}_{31} \mathrm{O}_{2}{ }^{120} \mathrm{Sn}[\mathrm{M}-\mathrm{Bu}]^{+} 411.1389$; found 411.1367.

(Z)-4-Tributylstannyl-1-(2,4,6-trimethoxy-phenyl)-but-2-en-1-ol (13d): According to the general procedure for hydrostannations $(Z)-\mathbf{1 3 d}$ was obtained from allenol $\mathbf{1 2 d}(83 \mathrm{mg}, 0.40 \mathrm{mmol})$ at $55^{\circ} \mathrm{C}$ as the only allylstannane isomer (42 $\mathrm{mg}, 0.08 \mathrm{mmol}, 20 \%$ ) besides the elimination product $\mathbf{1 4 d}$ (32 $\mathrm{mg}, 0.22 \mathrm{mmol}, 43 \%)$ and the regiosiomer 15d (39 mg, $0.07 \mathrm{mmol}, 19 \%)$. (Z)-13d: ${ }^{1} \mathrm{H}$ NMR (500 $\left.\mathrm{MHz}, \mathrm{CDCl}_{3}\right): \delta=0.80-0.88(\mathrm{~m}, 15 \mathrm{H}, 1-\mathrm{H}, 4-\mathrm{H}), 1.23-1.31$ (m, 6H, 3-H), 1.40-1.55 (m, 6H, 2-H), $1.59(\mathrm{~m}, 2 \mathrm{H}, 5-\mathrm{H}), 3.24\left(\mathrm{~d},{ }^{3} J_{\mathrm{OH}, 8}=11.0 \mathrm{~Hz}, 1 \mathrm{H}, \mathrm{OH}\right), 3.79-3.83(\mathrm{~m}, 9 \mathrm{H}, 13-\mathrm{H}, 14-\mathrm{H}), 5.55-5.68$ $(\mathrm{m}, 2 \mathrm{H}, 6-\mathrm{H}, 8-\mathrm{H}), 6.17(\mathrm{~m}, 1 \mathrm{H}, 7-\mathrm{H}), 6.11(\mathrm{~s}, 2 \mathrm{H}, 11-\mathrm{H}) ;{ }^{13} \mathrm{C} \mathrm{NMR}\left(125 \mathrm{MHz}, \mathrm{CDCl}_{3}\right): \delta=9.1(\mathrm{t}$, C-1), 9.8 (t, C-5), 13.7 (q, C-4), 27.8 (t, C-3), 29.0 (t, C-2), 55.2 (q, C-14), 55.5 (q, C-13), 63.0 (d, C8), 90.7 (d, C-11), 90.9 (s, C-9), 127.1 (d, C-6), 130.0 (d, C-7), 158.2 (s, C-12), 160.1 (s, C-10); HRMS $\left(\mathrm{FAB}^{+}\right)$: calcd. for $\mathrm{C}_{25} \mathrm{H}_{45} \mathrm{O}_{4} \mathrm{Sn}[\mathrm{M}+\mathrm{H}]^{+}$529.2340; found 529.2360.

(E/Z)-1-(2-Bromophenyl)-4-tributylstannylbut-2-en-1-ol (13e): According to the general procedure for hydrostannations 13e was obtained from allenol 12e $(128 \mathrm{mg}, 0.57 \mathrm{mmol})$ as a colorless liquid (253 mg, $0.49 \mathrm{mmol}, 86 \%)$ and a 4:3 (E/Z)-mixture. (E)-13e: ${ }^{1} \mathrm{H} \mathrm{NMR}(500 \mathrm{MHz}$, $\left.\mathrm{CDCl}_{3}\right): \delta=0.80-0.89(\mathrm{~m}, 15 \mathrm{H}, 1-\mathrm{H}, 4-\mathrm{H}), 1.21-1.30(\mathrm{~m}, 6 \mathrm{H}, 3-\mathrm{H}), 1.39-1.48(\mathrm{~m}, 6 \mathrm{H}, 2-\mathrm{H}), 1.73$ 
(m, 1H, OH), $1.92(\mathrm{~m}, 2 \mathrm{H}, 5-\mathrm{H}), 5.37(\mathrm{~m}, 1 \mathrm{H}, 7-\mathrm{H}), 5.48(\mathrm{~m}, 1 \mathrm{H}, 8-\mathrm{H}), 5.90(\mathrm{~m}, 1 \mathrm{H}, 6-\mathrm{H}), 7.10(\mathrm{~m}$, $1 \mathrm{H}, 10-\mathrm{H}), 7.31(\mathrm{~m}, 1 \mathrm{H}, 12-\mathrm{H}), 7.50(\mathrm{~m}, 1 \mathrm{H}, 11-\mathrm{H}), 7.56(\mathrm{~m}, 1 \mathrm{H}, 13-\mathrm{H}) ;{ }^{13} \mathrm{C}$ NMR $(125 \mathrm{MHz}$, $\mathrm{CDCl}_{3}$ ): $\delta=9.5$ (t, C-1), 13.6 (q, C-4), 14.6 (t, C-5), 27.3 (t, C-3), 29.0 (t, C-2), 74.1 (d, C-8), 122.3 (s, C-14), 124.7 (d, C-12), 127.5 (d, C-10), 128.3 (d, C-6), 128.5 (d, C-11), 132.6 (d, C-13), 133.9 (d, C-7), 142.8 (d, C-9); (Z)-13e (selected signals): ${ }^{1} \mathrm{H}$ NMR $\left(500 \mathrm{MHz}, \mathrm{CDCl}_{3}\right): \delta=5.34$ (m, 1H, 7-H), $5.78(\mathrm{~m}, 1 \mathrm{H}, 6-\mathrm{H}), 5.82(\mathrm{~m}, 1 \mathrm{H}, 8-\mathrm{H}) ;{ }^{13} \mathrm{C} \mathrm{NMR}\left(125 \mathrm{MHz}, \mathrm{CDCl}_{3}\right): \delta=9.3(\mathrm{t}, \mathrm{C}-1), 11.9(\mathrm{t}, \mathrm{C}-5)$, 13.7 (q, C-4), 29.1 (t, C-2), 69.1 (d, C-8), 122.7 (s, C-14), 124.6 (d, C-12), 127.6 (d, C-10), 127.8 (d, C-6), 128.8 (d, C-11), 132.8 (d, C-13), 133.2 (d, C-7), 143.1 (s, C-9); HRMS (CI): calcd. for $\mathrm{C}_{22} \mathrm{H}_{37}{ }^{79} \mathrm{BrO}^{120} \mathrm{Sn}[\mathrm{M}]^{+}$516.1013; found 516.1031; calcd. for $\mathrm{C}_{18} \mathrm{H}_{28}{ }^{79} \mathrm{BrO}^{120} \mathrm{Sn}[\mathrm{M}-\mathrm{Bu}]^{+} 459.0337$; found 459.0341; $\mathrm{C}_{22} \mathrm{H}_{37} \mathrm{BrOSn}$ (516.13): calcd. C 51.20, H 7.23; found C 50.88, H 6.96.

(E/Z)-1-(2-Chloro-phenyl)-4-tributylstannyl-but-2-en-1-ol (13f): According to the general procedure for hydrostannations $\mathbf{1 3 f}$ was obtained from allenol $\mathbf{1 2 f}(83 \mathrm{mg}, 0.50 \mathrm{mmol})$ as a colorless liquid (159 mg, $0.34 \mathrm{mmol}, 68 \%)$ and a 1:1 (E/Z)-mixture. $(Z)-13 f:{ }^{1} \mathrm{H}$ NMR $\left(500 \mathrm{MHz}, \mathrm{CDCl}_{3}\right): \delta=$ 0.80-0.88 (m, 15H, 1-H, 4-H), 1.23-1.31 (m, 6H, 3-H), 1.40-1.55 (m, 6H, 2-H), 1.70-1.88 (m, 3H, $5-\mathrm{H}, \mathrm{OH}), 5.30\left(\mathrm{dd},{ }^{3} J_{7,6}=10.4 \mathrm{~Hz},{ }^{3} J_{7,8}=8.8 \mathrm{~Hz},{ }^{4} J_{7, \mathrm{Sn}}=38.8 \mathrm{~Hz}, 1 \mathrm{H}, 7-\mathrm{H}\right) 5.47(\mathrm{~m}, 1 \mathrm{H}, 8-\mathrm{H}), 5.76$ (m, 1H, 6-H), 7.29-7.33 (m, 4H, 10-H, 11-H); ${ }^{13} \mathrm{C} \mathrm{NMR} \mathrm{(125} \mathrm{MHz,} \mathrm{CDCl} 3$ ): $\delta=9.4(\mathrm{t}, \mathrm{C}-1), 11.3(\mathrm{t}$, C-5), 13.6 (q, C-4), 27.3 (t, C-3), 29.0 (t, C-2), 68.8 (d, C-8), 125.6 (d, C-6), 127.2 (d, C-10), 127.3 (d, C-11), 132.5 (s, C-12), 132.7 (d, C-7), 142.3 (s, C-9); (E)-13f (selected signals): ${ }^{1} \mathrm{H}$ NMR (500 $\left.\mathrm{MHz}, \mathrm{CDCl}_{3}\right): \delta=5.09(\mathrm{~m}, 1 \mathrm{H}, 8-\mathrm{H}), 5.46\left(\mathrm{dd},{ }^{3} J_{7,6}=14.8 \mathrm{~Hz},{ }^{3} J_{7,8}=7.3 \mathrm{~Hz},{ }^{4} J_{7, \mathrm{Sn}}=41.9 \mathrm{~Hz}, 1 \mathrm{H}, 7-\right.$ $\mathrm{H}), 5.84(\mathrm{~m}, 1 \mathrm{H}, 6-\mathrm{H}) ;{ }^{13} \mathrm{C}$ NMR $\left(125 \mathrm{MHz}, \mathrm{CDCl}_{3}\right): \delta=14.5(\mathrm{t}, \mathrm{C}-5), 75.0(\mathrm{~d}, \mathrm{C}-8), 127.2(\mathrm{~d}, \mathrm{C}-6)$, 132.8 (d, C-7); $\mathrm{C}_{22} \mathrm{H}_{37} \mathrm{ClOSn}$ (471.70): calcd. C 56.01, H 7.91; found C 55.97, H 7.99.

(E/Z)-1-(2,6-Dichloro-phenyl)-4-tributylstannyl-but-2-en-1-ol (13g): According to the general procedure for hydrostannations $\mathbf{1 3 g}$ was obtained from allenol $\mathbf{1 2 g}(109 \mathrm{mg}, 0.50 \mathrm{mmol})$ as a colorless liquid (169 mg, $0.33 \mathrm{mmol}, 67 \%)$ and a 1:2 (E/Z)-mixture. (Z)-13k: ${ }^{1} \mathrm{H}$ NMR (500 MHz, $\left.\mathrm{CDCl}_{3}\right): \delta=0.80-0.88(\mathrm{~m}, 15 \mathrm{H}, 1-\mathrm{H}, 4-\mathrm{H}), 1.23-1.31(\mathrm{~m}, 6 \mathrm{H}, 3-\mathrm{H}), 1.40-1.55(\mathrm{~m}, 6 \mathrm{H}, 2-\mathrm{H}), 1.92$ $(\mathrm{m}, 2 \mathrm{H}, 5-\mathrm{H}), 2.63\left(\mathrm{~d},{ }^{3} J_{\mathrm{OH}, 8}=8.8 \mathrm{~Hz}, 1 \mathrm{H}, \mathrm{OH}\right), 5.70-5.89(\mathrm{~m}, 2 \mathrm{H}, 6-\mathrm{H}, 7-\mathrm{H}) 6.17(\mathrm{~m}, 1 \mathrm{H}, 8-\mathrm{H})$, 7.25-7.36 (m, 3H, 11-H, 12-H); ${ }^{13} \mathrm{C}$ NMR (125 MHz, $\left.\mathrm{CDCl}_{3}\right): \delta=8.1(\mathrm{t}, \mathrm{C}-1), 13.6$ (q, C-4), 14.7 (t, C-5), 27.2 (t, C-3), 29.0 (t, C-2), 67.8 (d, C-8), 123.0 (d, C-6), 129.1 (2d, C-11, C-12), 134.1 (d,s, C7, C-10), 138.4 (s, C-9); (E)-13k (selected signals): ${ }^{1} \mathrm{H}$ NMR (500 MHz, $\left.\mathrm{CDCl}_{3}\right): \delta=1.74$ (m, 2H, 5$\mathrm{H}), 2.90\left(\mathrm{~d},{ }^{3} \mathrm{~J}_{\mathrm{OH}, 8}=9.8 \mathrm{~Hz}, 1 \mathrm{H}, \mathrm{OH}\right) ;{ }^{13} \mathrm{C} \mathrm{NMR}\left(125 \mathrm{MHz}, \mathrm{CDCl}_{3}\right): \delta=14.7$ (t, C-5), $72.9(\mathrm{~d}, \mathrm{C}-8)$; $\mathrm{C}_{22} \mathrm{H}_{36} \mathrm{Cl}_{2} \mathrm{OSn}$ (506.14): calcd. C 52.21, H 7.17; found C 52.33, H 7.24.

(E/Z)-1-(2-Nitro-phenyl)-4-tributylstannyl-but-2-en-1-ol (13h): According to the general procedure for hydrostannations $\mathbf{1 3 h}$ was obtained from allenol $\mathbf{1 2 h}(156 \mathrm{mg}, 0.80 \mathrm{mmol})$ as a yellow 
liquid (297 mg, $0.60 \mathrm{mmol}, 76 \%)$ and a 1:1 (E/Z)-mixture. (E)-13h: ${ }^{1} \mathrm{H}$ NMR $\left(500 \mathrm{MHz}, \mathrm{CDCl}_{3}\right): \delta$ $=0.78-0.88(\mathrm{~m}, 15 \mathrm{H}, 1-\mathrm{H}, 4-\mathrm{H}), 1.23-1.32(\mathrm{~m}, 6 \mathrm{H}, 3-\mathrm{H}), 1.40-1.55(\mathrm{~m}, 6 \mathrm{H}, 2-\mathrm{H}), 1.65-1.91(\mathrm{~m}, 2 \mathrm{H}$, $5-\mathrm{H}), 2.19\left(\mathrm{~d},{ }^{3} J_{\mathrm{OH}, 8}=4.0 \mathrm{~Hz}, 1 \mathrm{H}, \mathrm{OH}\right), 5.45\left(\mathrm{dd},{ }^{3} J_{7,6}=15.2 \mathrm{~Hz},{ }^{3} J_{7,8}=7.0 \mathrm{~Hz},{ }^{4} J_{7, \mathrm{Sn}}=41.1 \mathrm{~Hz}, 1 \mathrm{H}\right.$, 7-H), 5.91 (m, 1H, 6-H), 5.97 (m, 1H, 8-H), 7.37-7.89 (m, 4H, 11-H, 12-H, 13-H, 14-H); ${ }^{13} \mathrm{C}$ NMR (125 MHz, $\mathrm{CDCl}_{3}$ ): $\delta=9.4$ (t, C-1), 13.6 (q, C-4), 14.6 (t, C-5), 27.3 (t, C-3), 29.1 (t, C-2), 70.5 (d, C-8), 124.4 (d, C-6), 125.1 (d, C-11), 128.3 (d, C-12), 128.8 (d, C-7), 133.2 (d, C-14), 134.5 (d, C13) 138.9 (s, C-9), 148.3 (s, C-10); (Z)-13h (selected signals): ${ }^{1} \mathrm{H}$ NMR (500 MHz, $\mathrm{CDCl}_{3}$ ): $\delta=5.35$ $\left(\mathrm{dd},{ }^{3} J_{7,6}=9.4 \mathrm{~Hz},{ }^{3} J_{7,8}=9.4 \mathrm{~Hz},{ }^{4} J_{7, \mathrm{Sn}}=38.7 \mathrm{~Hz}, 1 \mathrm{H}, 7-\mathrm{H}\right), 5.71(\mathrm{~m}, 1 \mathrm{H}, 8-\mathrm{H}), 5.82\left(\mathrm{dt},{ }^{3} J_{6,5}=9.8\right.$ $\left.\mathrm{Hz},{ }^{3} J_{6,7}=9.4 \mathrm{~Hz},{ }^{3} J_{6, \mathrm{Sn}}=49.3 \mathrm{~Hz}, 1 \mathrm{H}, 6-\mathrm{H}\right) ;{ }^{13} \mathrm{C} \mathrm{NMR}\left(125 \mathrm{MHz}, \mathrm{CDCl}_{3}\right): \delta=11.6(\mathrm{t}, \mathrm{C}-5), 65.6$ (d, C-8), 123.4 (d, C-6), 127.9 (d, C-7); $\mathrm{C}_{22} \mathrm{H}_{37} \mathrm{NO}_{3} \mathrm{Sn}$ (482.25): calcd. C 54.79, H 7.73, N 2.90; found C 54.78, H 7.71, N 2.80.

(E/Z)-1-(4-Nitrophenyl)-4-tributylstannylbut-2-en-1-ol (13i): According to the general procedure for hydrostannations $\mathbf{1 3 i}$ was obtained from allenol $\mathbf{1 2 i}$ (133 $\mathrm{mg}, 0.69 \mathrm{mmol})$ as a yellow liquid (218 $\mathrm{mg}, 0.58 \mathrm{mmol}, 84 \%)$ and a 4:3 (E/Z)-mixture. (E)-13i: ${ }^{1} \mathrm{H}$ NMR (500 MHz, $\left.\mathrm{CDCl}_{3}\right): \delta=0.81-0.90$ (m, 15H, 1-H, 4-H), 1.22-1.31 (m, 6H, 3-H), 1.40-1.49 (m, 6H, 2-H), 1.74-1.96 (m, 3H, 5-H, OH), $5.21\left(\mathrm{dd},{ }^{3} J_{8,7}=7.8 \mathrm{~Hz},{ }^{3} J_{8, \mathrm{OH}}=3.1 \mathrm{~Hz}, 1 \mathrm{H}, 8-\mathrm{H}\right), 5.35\left(\mathrm{dd},{ }^{3} J_{7,6}=14.9 \mathrm{~Hz},{ }^{3} J_{7,8}=7.8 \mathrm{~Hz},{ }^{4} J_{7, \mathrm{Sn}}=\right.$ $41.7 \mathrm{~Hz}, 1 \mathrm{H}, 7-\mathrm{H}), 5.93\left(\mathrm{dt},{ }^{3} J_{6,7}=14.9 \mathrm{~Hz},{ }^{3} J_{6,5}=8.5 \mathrm{~Hz},{ }^{3} J_{6, \mathrm{Sn}}=49.7 \mathrm{~Hz}, 1 \mathrm{H}, 6-\mathrm{H}\right), 7.50\left(\mathrm{~d},{ }^{3} J_{10,11}\right.$ $=8.6 \mathrm{~Hz}, 2 \mathrm{H}, 10-\mathrm{H}), 8.16\left(\mathrm{~d},{ }^{3} J_{11,10}=8.6 \mathrm{~Hz}, 2 \mathrm{H}, 11-\mathrm{H}\right) ;{ }^{13} \mathrm{C} \mathrm{NMR}\left(125 \mathrm{MHz}, \mathrm{CDCl}_{3}\right): \delta=9.4(\mathrm{t}, \mathrm{C}-$ 1), 13.6 (q, C-4), 14.7 (t, C-5), 27.3 (t, C-3), 29.1 (t, C-2), 75.0 (d, C-8), 123.5 (d, C-11), 126.4 (d, C6), 126.6 (d, C-10), 135.2 (d, C-7), 147.0 (s, C-9), 151.1 (s, C-12); (Z)-13i (selected signals): ${ }^{1} \mathrm{H}$ NMR (500 MHz, $\left.\mathrm{CDCl}_{3}\right): \delta=5.25(\mathrm{~m}, 1 \mathrm{H}, 7-\mathrm{H}), 5.60\left(\mathrm{dd},{ }^{3} J_{8,7}=8.8 \mathrm{~Hz},{ }^{3} J_{8, \mathrm{OH}}=3.4 \mathrm{~Hz}, 1 \mathrm{H}, 8-\mathrm{H}\right)$, $5.84(\mathrm{~m}, 1 \mathrm{H}, 6-\mathrm{H}), 7.55\left(\mathrm{~d},{ }^{3} J_{10,11}=8.6 \mathrm{~Hz}, 2 \mathrm{H}, 10-\mathrm{H}\right), 8.17\left(\mathrm{~d},{ }^{3} J_{11,10}=8.6 \mathrm{~Hz}, 2 \mathrm{H}, 11-\mathrm{H}\right) ;{ }^{13} \mathrm{C} \mathrm{NMR}$ (125 MHz, $\mathrm{CDCl}_{3}$ ): $\delta=9.5$ (t, C-1), 11.5 (t, C-5), 68.6 (d, C-8), 123.6 (d, C-11), 124.9 (d, C-6), 126.7 (d, C-10), 134.0 (d, C-7), 151.4 (s, C-12); HRMS (CI): calcd. for $\mathrm{C}_{22} \mathrm{H}_{36} \mathrm{NO}_{3}{ }^{120} \mathrm{Sn}[\mathrm{M}]^{+}$ 482.1695; found 482.1706; calcd. for $\mathrm{C}_{18} \mathrm{H}_{27} \mathrm{NO}_{3}{ }^{120} \mathrm{Sn}[\mathrm{M}-\mathrm{Bu}]^{+} 425.1005$; found 425.1009; $\mathrm{C}_{22} \mathrm{H}_{37} \mathrm{NO}_{3} \mathrm{Sn}$ (482.24): calcd. C 54.80, H 7.73, N 2.90; found C 54.79, H 7.68, N 3.22.

(E/Z)-1-Phenyl-5-tributylstannylpent-3-en-2-ol (13k): According to the general procedure for hydrostannations 13k was obtained from allenol 12k $(218 \mathrm{mg}, 1.36 \mathrm{mmol})$ as a colorless liquid (883 $\mathrm{mg}, 1.96 \mathrm{mmol}, 68 \%)$ and a 2:3 (E/Z)-mixture. (Z)-13k: ${ }^{1} \mathrm{H}$ NMR $\left(500 \mathrm{MHz}, \mathrm{CDCl}_{3}\right): \delta=0.81-0.90$ $(\mathrm{m}, 15 \mathrm{H}, 1-\mathrm{H}, 4-\mathrm{H}), 1.25-1.32(\mathrm{~m}, 6 \mathrm{H}, 3-\mathrm{H}), 1.40\left(\mathrm{~d},{ }^{3} \mathrm{~J}_{\mathrm{OH}, 8}=3.8 \mathrm{~Hz}, 1 \mathrm{H}, \mathrm{OH}\right) 1.42-1.50$ (m, 6H, 2H), 1.67-1.80 (m, 2H, 5-H), $2.79(\mathrm{~m}, 2 \mathrm{H}, 9-\mathrm{H}), 4.63(\mathrm{~m}, 1 \mathrm{H}, 8-\mathrm{H}), 5.71(\mathrm{~m}, 1 \mathrm{H}, 7-\mathrm{H}), 5.68(\mathrm{~m}, 1 \mathrm{H}$, 6-H), 7.20-7.31 (m, 5H, 11-H, 12-H, 13-H); $\left.{ }^{13} \mathrm{C} \mathrm{NMR} \mathrm{(125} \mathrm{MHz,} \mathrm{CDCl}_{3}\right): \delta=9.4$ (t, C-1), $11.2(\mathrm{t}$, C-5), 13.7 (q, C-4), 27.3 (t, C-3), 29.1 (t, C-2), 44.0 (t, C-9), 68.3 (d, C-8), 126.4 (d, C-6), 127.2 (d, C-11), 128.4 (d, C-13), 129.6 (d, C-12), 132.1 (d, C-7), 138.3 (s, C-10); (E)-13k (selected signals): 
${ }^{1} \mathrm{H}$ NMR $\left(500 \mathrm{MHz}, \mathrm{CDCl}_{3}\right): \delta=4.26(\mathrm{~m}, 1 \mathrm{H}, 8-\mathrm{H}), 5.33\left(\mathrm{dd},{ }^{3} J_{7,6}=15.1 \mathrm{~Hz},{ }^{3} J_{7,8}=7.3 \mathrm{~Hz},{ }^{4} J_{7, \mathrm{Sn}}=\right.$ $41.6 \mathrm{~Hz}, 1 \mathrm{H}, 7-\mathrm{H}), 5.80\left(\mathrm{dt},{ }^{3} J_{6,7}=15.1 \mathrm{~Hz},{ }^{3} J_{6,5}=8.8 \mathrm{~Hz},{ }^{3} J_{6, \mathrm{Sn}}=54.2 \mathrm{~Hz}, 1 \mathrm{H}, 6-\mathrm{H}\right) ;{ }^{13} \mathrm{C} \mathrm{NMR}(125$ $\mathrm{MHz}, \mathrm{CDCl}_{3}$ ): $\delta=9.2$ (t, C-1), 14.3 (t, C-5), 44.4 (t, C-9), 74.2 (d, C-8), 125.9 (d, C-11), 126.3 (d, C6), 129.5 (d, C-12), 132.6 (d, C-7), 138.4 (s, C-10); HRMS (CI): calcd. for $\mathrm{C}_{19} \mathrm{H}_{31} \mathrm{O}^{120} \mathrm{Sn}[\mathrm{M}-\mathrm{Bu}]^{+}$ 395.1371; found 395.1384.

(E/Z)-6-Methyl-1-tributylstannylhept-2-en-4-ol (131): According to the general procedure for hydrostannations $13 \mathrm{l}$ was obtained from allenol $\mathbf{1 2 l}(190 \mathrm{mg}, 1.51 \mathrm{mmol})$ as a colorless liquid (398 mg, 0.96 mmol, 63\%) and a 3:5 (E/Z)-mixture. (Z)-13I: ${ }^{1} \mathrm{H}$ NMR (500 MHz, $\left.\mathrm{CDCl}_{3}\right): \delta=0.84-0.94$ (m, 21H, 1-H, 4-H, 11-H), 1.17 (bs, 1H, OH), 1.21 (m, 1H, 9-H), 1.25-1.33 (m, 6H, 3-H), 1.41-1.53 (m, 7H, 2-H, 9'-H), 1.64-1.83 (m, 3H, 5-H, 10-H), 4.49 (m, 1H, 8-H), 5.09 (dd, ${ }^{3} J_{7,6}=10.4 \mathrm{~Hz},{ }^{3} J_{7,8}$ $=8.8 \mathrm{~Hz}, 1 \mathrm{H}, 7-\mathrm{H}), 5.63(\mathrm{~m}, 1 \mathrm{H}, 6-\mathrm{H}) ;{ }^{13} \mathrm{C} \mathrm{NMR}\left(125 \mathrm{MHz}, \mathrm{CDCl}_{3}\right): \delta=9.3(\mathrm{t}, \mathrm{C}-1), 11.1(\mathrm{t}, \mathrm{C}-5)$, 13.7 (q, C-4), 22.7 (2q, C-11), 23.4 (d, C-10), 27.3 (t, C-3), 29.1 (t, C-2), 46.7 (t, C-9), 65.5 (d, C-8), 127.6 (d, C-6), 131.4 (d, C-7); (E)-13I (selected signals): ${ }^{1} \mathrm{H}$ NMR (500 MHz, $\left.\mathrm{CDCl}_{3}\right): \delta=4.06$ (m, $1 \mathrm{H}, 8-\mathrm{H}), 5.24\left(\mathrm{dddd},{ }^{3} J_{7,6}=15.1 \mathrm{~Hz},{ }^{3} J_{7,8}=7.9 \mathrm{~Hz},{ }^{4} J_{7,5}=0.9 \mathrm{~Hz},{ }^{4} J_{7,5}=1.3 \mathrm{~Hz}, 1 \mathrm{H}, 7-\mathrm{H}\right), 5.78$ $\left(\mathrm{dtd},{ }^{3} J_{6,7}=15.1 \mathrm{~Hz},{ }^{3} J_{6,5}=8.8 \mathrm{~Hz},{ }^{4} J_{6,8}=0.6 \mathrm{~Hz},{ }^{3} J_{6, \mathrm{Sn}}=49.5 \mathrm{~Hz}, 1 \mathrm{H}, 6-\mathrm{H}\right) ;{ }^{13} \mathrm{C} \mathrm{NMR}(125 \mathrm{MHz}$, $\mathrm{CDCl}_{3}$ ): $\delta=9.2$ (t, C-4), 14.2 (t, C-5), 72.0 (d, C-8), 128.7 (d, C-6), 132.2 (d, C-7); HRMS (CI): calcd. for $\mathrm{C}_{16} \mathrm{H}_{33} \mathrm{O}^{120} \mathrm{Sn}[\mathrm{M}-\mathrm{Bu}]^{+} 361.1535$; found 361.1544 .

(E/Z)-1-(Cyclohex-3-en-1-yl)-4-tributylstannylbut-2-en-1-ol (13m): According to the general procedure for hydrostannations $\mathbf{1 3 m}$ was obtained from allenol $\mathbf{1 2 m}(225 \mathrm{mg}, 1.50 \mathrm{mmol})$ as a colorless liquid (512 mg, $1.13 \mathrm{mmol}, 75 \%)$ and a 2:3 diastereomeric $(E / Z)$-mixture. $(Z)-\mathbf{1 3 m}:{ }^{1} \mathrm{H}$ NMR (500 MHz, $\left.\mathrm{CDCl}_{3}\right): \delta=0.84-0.93(\mathrm{~m}, 15 \mathrm{H}, 1-\mathrm{H}, 4-\mathrm{H}), 1.18(\mathrm{~m}, 1 \mathrm{H}, \mathrm{OH}), 1.27-1.33(\mathrm{~m}, 6 \mathrm{H}$, 3-H), 1.44-1.51 (m, 6H, 2-H), 1.65 (m, 1H, 14-H), 1.63-2.18 (m, 8H, 5-H, 9-H, 10-H, 13-H, 14'-H), $3.80(\mathrm{~m}, 1 \mathrm{H}, 8-\mathrm{H}), 5.29(\mathrm{~m}, 1 \mathrm{H}, 7-\mathrm{H}), 5.65-5.69(\mathrm{~m}, 2 \mathrm{H}, 11-\mathrm{H}, 12-\mathrm{H}), 5.78(\mathrm{~m}, 1 \mathrm{H}, 6-\mathrm{H}) ;{ }^{13} \mathrm{C} \mathrm{NMR}$ (125 MHz, $\mathrm{CDCl}_{3}$ ): $\delta=9.4$ (t, C-1), 11.3 (t, C-5), 13.7 (q, C-4), 24.8 (t, C-14), 24.9 (t, C-14'), 25.1 (t, C-13), 27.3 (t, C-3), 27.5 (t, C-10), 27.9 (t, C-10'), 29.1 (t, C-2), 40.0 (d, C-9), 40.2 (d, C-9'), 70.5 (d, C-8), 71.1 (d, C-8'), 125.4 (d, C-11), 125.5 (d, C-11'), 126.4 (d, C-6), 126.5 (d, C-6'), 127.1 (d, C-12), 127.2 (d, C-12'), 132.7 (d, C-7), 132.8 (d, C-7'); (E)-13m (selected signals): ${ }^{1} \mathrm{H}$ NMR (500 $\left.\mathrm{MHz}, \mathrm{CDCl}_{3}\right): \delta=4.23(\mathrm{~m}, 1 \mathrm{H}, 8-\mathrm{H}), 5.15(\mathrm{~m}, 1 \mathrm{H}, 7-\mathrm{H}), 5.73(\mathrm{~m}, 1 \mathrm{H}, 6-\mathrm{H}) ;{ }^{13} \mathrm{C} \mathrm{NMR}(125 \mathrm{MHz}$, $\left.\mathrm{CDCl}_{3}\right): \delta=9.3(\mathrm{t}, \mathrm{C}-1), 14.4$ (t, C-5), 24.4 (t, C-13), 24.7 (t, C-14), 24.8 (t, C-14'), 27.3 (t, C-3), 27.7 (t, C-10), 27.9 (t, C-10'), 29.1 (t, C-2), 39.8 (d, C-9), 39.9 (d, C-9’), 77.5 (d, C-8), 77.7 (d, C8'), 126.2 (d, C-6), 126.6 (d, C-11), 126.7 (d, C-11'), 126.8 (d, C-12), 126.9 (d, C-12'), 133.4 (d, C7), 133.7 (d, C-7'); HRMS (CI): calcd. for $\mathrm{C}_{18} \mathrm{H}_{33} \mathrm{OSn}$ [M-Bu] ${ }^{+}$385.1469; found 385.1471. 
1-Phenylbuta-1,3-diene (14a): ${ }^{1}$ Diene 14a was obtained as side product in the hydrostannation of allenol 12a at higher temperatures. ${ }^{1} \mathrm{H}$ NMR $\left(500 \mathrm{MHz}, \mathrm{CDCl}_{3}\right): \delta=5.17\left(\mathrm{dd},{ }^{3} J_{1(\text { trans }), 2}=10.1 \mathrm{~Hz}\right.$, $\left.{ }^{2} J_{1(\text { trans }), 1(\text { cis })}=0.6 \mathrm{~Hz}, 1 \mathrm{H}, 1-\mathrm{H}_{\text {trans }}\right), 5.33\left(\mathrm{dd},{ }^{3} J_{1(\text { cis }), 2}=17.7 \mathrm{~Hz},{ }^{2} J_{1(\text { cis }), 1(\text { trans })}=0.6 \mathrm{~Hz}, 1 \mathrm{H}, 1-\mathrm{H}_{\text {cis }}\right)$, $6.50(\mathrm{~m}, 1 \mathrm{H}, 2-\mathrm{H}), 6.56\left(\mathrm{~d},{ }^{3} J_{4,3}=15.6 \mathrm{~Hz}, 1 \mathrm{H}, 4-\mathrm{H}\right), 6.78\left(\mathrm{dd},{ }^{3} J_{3,4}=15.6 \mathrm{~Hz},{ }^{3} J_{3,2}=10.4 \mathrm{~Hz}, 1 \mathrm{H}, 3-\right.$ $\mathrm{H}), 7.23(\mathrm{~m}, 1 \mathrm{H}, 8-\mathrm{H}), 7.31(\mathrm{~m}, 2 \mathrm{H}, 7-\mathrm{H}), 7.40(\mathrm{~m}, 2 \mathrm{H}, 6-\mathrm{H}) ;{ }^{13} \mathrm{C} \mathrm{NMR}\left(125 \mathrm{MHz}, \mathrm{CDCl}_{3}\right): \delta=$ 117.6 (t, C-1), 126.4 (d, C-8), 127.6 (d, C-3), 128.6 (d, C-6, C-7), 129.6 (d, C-4), 132.9 (d, C-2), $137.2(\mathrm{~s}, \mathrm{C}-5)$.

3-Tributylstannanyl-1-(2,4,6-trimethoxy-phenyl)-but-3-en-1-ol (15d): The vinylstannane 15d was obtained as a side product in the hydrostannation of allene 12d. ${ }^{1} \mathrm{H}$ NMR $\left(500 \mathrm{MHz}, \mathrm{CDCl}_{3}\right): \delta=$ 0.80-0.88 (m, 15H, 1-H, 4-H), 1.23-1.31 (m, 6H, 2-H), 1.40-1.55 (m, 6H, 4-H), $2.52\left(\mathrm{~m}, 1 \mathrm{H}, 7-\mathrm{H}_{\mathrm{a}}\right)$, $2.82\left(\mathrm{~m}, 1 \mathrm{H}, 7-\mathrm{H}_{\mathrm{b}}\right), 3.45\left(\mathrm{~d},{ }^{3} J_{\mathrm{OH}, 8}=11.3 \mathrm{~Hz}, 1 \mathrm{H}, \mathrm{OH}\right), 3.79-3.83(\mathrm{~m}, 9 \mathrm{H}, 13-\mathrm{H}, 14-\mathrm{H}), 5.01(\mathrm{~m}, 1 \mathrm{H}$, $8-\mathrm{H}), 5.20\left(\mathrm{dm},{ }^{3} J_{5 \mathrm{a}, \mathrm{Sn}}=61.8 \mathrm{~Hz}, 1 \mathrm{H}, 5-\mathrm{H}_{\mathrm{a}}\right), 5.71\left(\mathrm{dm},{ }^{3} J_{5 \mathrm{~b}, \mathrm{Sn}}=143.5 \mathrm{~Hz}, 1 \mathrm{H}, 5-\mathrm{H}_{\mathrm{b}}\right), 6.11(\mathrm{~s}, 2 \mathrm{H}, 11-$ $\mathrm{H}) ;{ }^{13} \mathrm{C}$ NMR (125 MHz, CDCl $)$ ): $\delta=8.4(\mathrm{t}, \mathrm{C}-1), 11.1$ (q, C-4), 27.4 (t, C-3), $29.2(\mathrm{t}, \mathrm{C}-2), 49.0(\mathrm{t}$, C-7), 55.2 (q, C-13), 55.7 (q, C-14), 67.3 (d, C-8), 90.7 (d, C-11), 90.9 (s, C-9), 126.9 (t, C-5), 153.1(s, C-6), 158.2 (s, C-12), 160.1 (s, C-10); HRMS $\left(\mathrm{FAB}^{+}\right)$: calcd. for $\mathrm{C}_{25} \mathrm{H}_{45} \mathrm{O}_{4} \mathrm{Sn}[\mathrm{M}+\mathrm{H}]$ 529.2340; found 529.2360.

(E)-4-Iodo-1-phenylbut-2-en-1-ol (16a): ${ }^{1} \mathrm{H}$ NMR $\left(500 \mathrm{MHz}, \mathrm{CDCl}_{3}\right): \delta=2.00\left(\mathrm{~d},{ }^{3} \mathrm{~J}_{\mathrm{OH}, 4}=3.8 \mathrm{~Hz}\right.$, $1 \mathrm{H}, \mathrm{OH}), 3.88\left(\mathrm{~d},{ }^{3} J_{1,2}=8.2 \mathrm{~Hz}, 2 \mathrm{H}, 1-\mathrm{H}\right), 5.20(\mathrm{~m}, 1 \mathrm{H}, 4-\mathrm{H}), 5.88\left(\mathrm{dd},{ }^{3} J_{3,2}=15.3 \mathrm{~Hz},{ }^{3} J_{3,4}=6.0\right.$ $\mathrm{Hz}, 1 \mathrm{H}, 3-\mathrm{H}), 6.04\left(\mathrm{dtd},{ }^{3} J_{2,3}=15.3 \mathrm{~Hz},{ }^{3} J_{2,1}=8.2 \mathrm{~Hz},{ }^{4} J_{2,4}=0.9 \mathrm{~Hz}, 1 \mathrm{H}, 2-\mathrm{H}\right), 7.22-7.40$ (m, 5H, 6$\mathrm{H}, 7-\mathrm{H}, 8-\mathrm{H}) ;{ }^{13} \mathrm{C}$ NMR (125 MHz, $\mathrm{CDCl}_{3}$ ): $\delta=4.4$ (t, C-1), 73.8 (d, C-4), 126.3 (d, C-2), 127.9 (s, C-6, C-8), 128.7 (d, C-7), 135.5 (d, C-3), 142.3 (s, C-5).

(E)-4-Iodo-1-(2-nitro-phenyl)-but-2-en-1-ol (16h): According to the preparation of 16a iodide 16h was obtained from allylstannane $13 \mathrm{~h}$ (48 $\mathrm{mg}, 0.10 \mathrm{mmol}$ ) and iodine (40 mg, $0.20 \mathrm{mmol}$ ) as a rather unstable yellow liquid $(20 \mathrm{mg}, 0.07 \mathrm{mmol})$ and as a single isomer. ${ }^{1} \mathrm{H}$ NMR $\left(500 \mathrm{MHz}, \mathrm{CDCl}_{3}\right): \delta=$ $2.60\left(\mathrm{~d},{ }^{3} J_{\mathrm{OH}, 4}=4.4 \mathrm{~Hz}, 1 \mathrm{H}, \mathrm{OH}\right), 3.86\left(\mathrm{~d},{ }^{3} J_{1,2}=7.9 \mathrm{~Hz}, 2 \mathrm{H}, 1-\mathrm{H}\right), 5.80(\mathrm{~m}, 1 \mathrm{H}, 4-\mathrm{H}), 5.89\left(\mathrm{dd},{ }^{3} J_{3,2}\right.$ $\left.=15.1 \mathrm{~Hz},{ }^{3} J_{3,4}=5.4 \mathrm{~Hz}, 1 \mathrm{H}, 3-\mathrm{H}\right), 6.11(\mathrm{~m}, 1 \mathrm{H}, 2-\mathrm{H}), 7.43-8.12(\mathrm{~m}, 4 \mathrm{H}, 7-\mathrm{H}, 8-\mathrm{H}, 9-\mathrm{H}, 10-\mathrm{H}) ;{ }^{13} \mathrm{C}$ NMR (125 MHz, CDCl 3 ): $\delta=3.9$ (t, C-1), 68.7 (d, C-4), 124.6 (d, C-7), 128.6 (d, C-2), 128.7 (d, C8), 129.6 (d, C-9), 133.1 (d, C-3), 133.7 (d, C-10) 137.3 (s, C-5), 148.1 (s, C-6).

(E)-4-Iodo-1-(4-nitrophenyl)-but-2-en-1-ol (16i): According to the preparation of 16a iodide 16i was obtained from allylstannane $13 \mathbf{i}(270 \mathrm{mg}, 0.56 \mathrm{mmol})$ and iodine $(152 \mathrm{mg}, 0.60 \mathrm{mmol})$ as a rather unstable yellow liquid (112 mg, $0.35 \mathrm{mmol}, 63 \%)$ and as a single isomer. ${ }^{1} \mathrm{H}$ NMR (500 MHz,

${ }^{1}$ Lebel, H.; Paquet, V. J. Am. Chem. Soc. 2004, 126, 320-328. 
$\left.\mathrm{CDCl}_{3}\right): \delta=2.18(\mathrm{bs}, 1 \mathrm{H}, \mathrm{OH}), 3.86\left(\mathrm{~d},{ }^{3} J_{1,2}=8.2 \mathrm{~Hz}, 2 \mathrm{H}, 1-\mathrm{H}\right), 5.32\left(\mathrm{~d},{ }^{3} J_{4,3}=6.5 \mathrm{~Hz}, 1 \mathrm{H}, 4-\mathrm{H}\right)$, $5.80\left(\mathrm{dd},{ }^{3} J_{3,2}=15.1 \mathrm{~Hz},{ }^{3} J_{3,4}=6.5 \mathrm{~Hz}, 1 \mathrm{H}, 3-\mathrm{H}\right), 6.09\left(\mathrm{dt},{ }^{3} J_{2,3}=15.1 \mathrm{~Hz},{ }^{3} J_{2,1}=8.2 \mathrm{~Hz}, 1 \mathrm{H}, 2-\mathrm{H}\right)$, $7.52(\mathrm{~m}, 2 \mathrm{H}, 6-\mathrm{H}), 8.20(\mathrm{~m}, 2 \mathrm{H}, 7-\mathrm{H}) ;{ }^{13} \mathrm{C} \mathrm{NMR}\left(125 \mathrm{MHz}, \mathrm{CDCl}_{3}\right): \delta=3.3(\mathrm{t}, \mathrm{C}-1), 73.1(\mathrm{~d}, \mathrm{C}-4)$, 123.8 (d, C-7), 126.9 (d, C-6), 130.4 (d, C-2), 134.1 (d, C-3), 147.5 (s, C-5), 149.2 (s, C-8).

cis/trans-2-(4-Methylphenyl)-3-vinyloxiran (17b): According to the general procedure for vinyl epoxide formation $\mathbf{1 7 b}$ was obtained from stannane $\mathbf{1 3 b}$ (990 mg, $2.19 \mathrm{mmol}$ ), iodine (583 mg, 2.30 $\mathrm{mmol}$ ) and $\mathrm{NaH}$ (106 mg, $4.40 \mathrm{mmol}$ ) as a colorless liquid (168 mg, $1.05 \mathrm{mmol}, 48 \%$ ) and a 1:3 cis/trans-mixture. trans-17b: ${ }^{1} \mathrm{H}$ NMR $\left(500 \mathrm{MHz}, \mathrm{CDCl}_{3}\right): \delta=3.24(\mathrm{~s}, 3 \mathrm{H}, 9-\mathrm{H}), 3.34\left(\mathrm{dd},{ }^{3} J_{3,2}=7.3\right.$ $\left.\mathrm{Hz},{ }^{3} J_{3,4}=1.8 \mathrm{~Hz}, 1 \mathrm{H}, 3-\mathrm{H}\right), 3.73\left(\mathrm{~d},{ }^{3} J_{4,3}=1.8 \mathrm{~Hz}, 1 \mathrm{H}, 4-\mathrm{H}\right), 5.32\left(\mathrm{dd},{ }^{3} J_{1(\text { trans }), 2}=10.4 \mathrm{~Hz}\right.$, $\left.{ }^{2} J_{1(\text { trans }), 1(\text { cis })}=0.9 \mathrm{~Hz}, 1 \mathrm{H}, 1-\mathrm{H}_{\text {trans }}\right), 5.50\left(\mathrm{dd},{ }^{3} J_{1(\text { cis }), 2}=17.4 \mathrm{~Hz},{ }^{2} J_{1(\text { cis }), 1(\text { trans })}=0.9 \mathrm{~Hz}, 1 \mathrm{H}, 1-\mathrm{H}_{\text {cis }}\right)$, $5.72\left(\mathrm{ddd},{ }^{3} J_{2,1(\text { cis })}=17.4 \mathrm{~Hz},{ }^{3} J_{2,1(\text { trans })}=10.4 \mathrm{~Hz},{ }^{3} J_{2,3}=7.3 \mathrm{~Hz}, 1 \mathrm{H}, 2-\mathrm{H}\right), 7.14-7.24(\mathrm{~m}, 4 \mathrm{H}, 6-\mathrm{H}, 7-$ $\mathrm{H}) ;{ }^{13} \mathrm{C}$ NMR $\left(125 \mathrm{MHz}, \mathrm{CDCl}_{3}\right): \delta=21.2$ (q, C-9), 60.2 (d, C-4), 62.8 (d, C-3), 119.4 (t, C-1), 125.4 (d, C-7), 129.2 (d, C-6), 132.2 (s, C-8), 135.2 (d, C-2), 138.0 (s, C-5); cis-17b: ${ }^{1} \mathrm{H}$ NMR (500 MHz, $\left.\mathrm{CDCl}_{3}\right): \delta=3.24(\mathrm{~s}, 3 \mathrm{H}, 9-\mathrm{H}), 3.63\left(\mathrm{dd},{ }^{3} J_{3,2}=8.2 \mathrm{~Hz},{ }^{3} J_{3,4}=4.3 \mathrm{~Hz}, 1 \mathrm{H}, 3-\mathrm{H}\right), 4.20\left(\mathrm{~d},{ }^{3} J_{4,3}=4.3\right.$ $\mathrm{Hz}, 1 \mathrm{H}, 4-\mathrm{H}), 5.26\left(\mathrm{dd},{ }^{3} J_{1(\text { trans }), 2}=10.4 \mathrm{~Hz},{ }^{2} J_{1(\text { trans }), 1(\text { cis })}=1.8 \mathrm{~Hz}, 1 \mathrm{H}, 1-\mathrm{H}_{\text {trans }}\right), 5.40\left(\mathrm{ddd},{ }^{3} J_{2,1(\text { cis })}=\right.$ $\left.17.1 \mathrm{~Hz},{ }^{3} J_{2,1(\text { trans })}=10.4 \mathrm{~Hz},{ }^{3} J_{2,3}=8.2 \mathrm{~Hz}, 1 \mathrm{H}, 2-\mathrm{H}\right), 5.53\left(\mathrm{dd},{ }^{3} J_{1(\text { cis }), 2}=17.1 \mathrm{~Hz},{ }^{2} J_{1(\text { cis }), 1(\text { trans })}=1.8\right.$ $\mathrm{Hz}, 1 \mathrm{H}, 1-\mathrm{H}_{\text {cis }}$ ), 7.14-7.24 (m, 4H, 6-H, 7-H); ${ }^{13} \mathrm{C} \mathrm{NMR} \mathrm{(125} \mathrm{MHz,} \mathrm{CDCl}_{3}$ ): $\delta=21.2$ (q, C-9), 58.8 (d, C-4), 59.8 (d, C-3), 121.7 (t, C-1), 126.3 (d, C-7), 128.8 (d, C-6), 132.0 (s, C-8), 135.9 (d, C-2), 137.4 (s, C-5); HRMS (CI): calcd. for $\mathrm{C}_{11} \mathrm{H}_{13} \mathrm{O}[\mathrm{M}+\mathrm{H}]^{+} 161.0968$; found 161.0967.

cis/trans-2-(2-Methoxyphenyl)-3-vinyloxiran (17c): According to the general procedure for vinyl epoxide formation 17c was obtained from stannane 13c (905 mg, $1.94 \mathrm{mmol})$, iodine (515 mg, 2.03 $\mathrm{mmol}$ ) and $\mathrm{NaH}(93 \mathrm{mg}, 3.88 \mathrm{mmol})$ as a colorless liquid (226 mg, $1.28 \mathrm{mmol}, 66 \%$ ) and a 1:2 cis/trans-mixture. trans-17c: ${ }^{1} \mathrm{H}$ NMR $\left(500 \mathrm{MHz}, \mathrm{CDCl}_{3}\right): \delta=3.29\left(\mathrm{dd},{ }^{3} J_{3,2}=7.3 \mathrm{~Hz},{ }^{3} J_{3,4}=1.8 \mathrm{~Hz}\right.$, $1 \mathrm{H}, 3-\mathrm{H}), 3.85(\mathrm{~s}, 3 \mathrm{H}, 11-\mathrm{H}), 4.15\left(\mathrm{~d},{ }^{3} J_{4,3}=1.8 \mathrm{~Hz}, 1 \mathrm{H}, 4-\mathrm{H}\right), 5.34\left(\mathrm{dd},{ }^{3} J_{1(\text { trans }), 2}=10.4 \mathrm{~Hz}\right.$, $\left.{ }^{2} J_{1(\text { trans }), 1(\text { cis })}=1.8 \mathrm{~Hz}, 1 \mathrm{H}, 1-\mathrm{H}_{\text {trans }}\right), 5.53\left(\mathrm{~d},{ }^{3} J_{1(\text { cis }), 2}=17.4 \mathrm{~Hz}, 1 \mathrm{H}, 1-\mathrm{H}_{\text {cis }}\right), 5.78\left(\mathrm{ddd},{ }^{3} J_{2,1(\text { cis })}=17.4\right.$ $\left.\mathrm{Hz},{ }^{3} J_{2,1 \text { (trans) }}=10.4 \mathrm{~Hz},{ }^{3} J_{2,3}=7.3 \mathrm{~Hz}, 1 \mathrm{H}, 2-\mathrm{H}\right), 6.87(\mathrm{~m}, 1 \mathrm{H}, 7-\mathrm{H}) .6 .96(\mathrm{~m}, 1 \mathrm{H}, 9-\mathrm{H}), 7.18\left(\mathrm{dd},{ }^{3} J_{6,7}\right.$ $\left.=9.1 \mathrm{~Hz},{ }^{4} J_{6,8}=1.8 \mathrm{~Hz}, 1 \mathrm{H}, 6-\mathrm{H}\right), 7.27(\mathrm{~m}, 1 \mathrm{H}, 8-\mathrm{H}) ;{ }^{13} \mathrm{C} \mathrm{NMR}\left(125 \mathrm{MHz}, \mathrm{CDCl}_{3}\right): \delta=55.3(\mathrm{q}, \mathrm{C}-$ 11), 55.8 (d, C-4), 62.3 (d, C-3), 110.1 (d, C-9), 119.3 (t, C-1), 120.6 (d, C-7), 124.9 (d, C-6), 128.6 (s, C-5), 128.8 (d, C-8), 135.4 (d, C-2), 157.9 (s, C-10); cis-17c: ${ }^{1} \mathrm{H}$ NMR (500 MHz, $\left.\mathrm{CDCl}_{3}\right): \delta=$ $3.72\left(\mathrm{dd},{ }^{3} J_{3,2}=8.2 \mathrm{~Hz},{ }^{3} J_{3,4}=4.3 \mathrm{~Hz}, 1 \mathrm{H}, 3-\mathrm{H}\right), 3.83(\mathrm{~s}, 3 \mathrm{H}, 11-\mathrm{H}), 4.38\left(\mathrm{~d},{ }^{3} J_{4,3}=4.3 \mathrm{~Hz}, 1 \mathrm{H}, 4-\mathrm{H}\right)$, $5.24\left(\mathrm{dd},{ }^{3} J_{1 \text { (trans) }, 2}=11.0 \mathrm{~Hz},{ }^{2} J_{1(\text { trans }), 1(\text { cis })}=1.2 \mathrm{~Hz}, 1 \mathrm{H}, 1-\mathrm{H}_{\text {trans }}\right), 5.36\left(\mathrm{ddd},{ }^{3} J_{2,1(\text { cis })}=17.4 \mathrm{~Hz}\right.$, $\left.{ }^{3} J_{2,1 \text { (trans })}=11.0 \mathrm{~Hz},{ }^{3} J_{2,3}=8.2 \mathrm{~Hz}, 1 \mathrm{H}, 2-\mathrm{H}\right), 5.53\left(\mathrm{~d},{ }^{3} J_{1(\text { cis }), 2}=17.4 \mathrm{~Hz}, 1 \mathrm{H}, 1-\mathrm{H}_{\text {cis }}\right), 6.87(\mathrm{~m}, 1 \mathrm{H}, 7-$ $\mathrm{H}), 6.96(\mathrm{~m}, 1 \mathrm{H}, 9-\mathrm{H}), 7.27(\mathrm{~m}, 1 \mathrm{H}, 8-\mathrm{H}), 7.33\left(\mathrm{dd},{ }^{3} J_{6,7}=7.3 \mathrm{~Hz},{ }^{4} J_{6,8}=1.2 \mathrm{~Hz}, 1 \mathrm{H}, 6-\mathrm{H}\right) ;{ }^{13} \mathrm{C} \mathrm{NMR}$ (125 MHz, $\mathrm{CDCl}_{3}$ ): $\delta=55.3$ (q, C-11), 56.0 (d, C-4), 59.3 (d, C-3), 109.9 (d, C-9), 120.0 (t, C-1), 
121.3 (d, C-7), 125.5 (d, C-6), 127.1 (d, C-8), 127.6 (s, C-5), 132.3 (d, C-2), 157.8 (s, C-10); HRMS (CI): calcd. for $\mathrm{C}_{11} \mathrm{H}_{12} \mathrm{O}_{2}[\mathrm{M}+\mathrm{H}]^{+}$177.0917; found 177.0916.

cis/trans-2-(2-Bromophenyl)-3-vinyloxiran (17e): According to the general procedure for vinyl epoxide formation 17e was obtained from stannane 13e (740 mg, $1.43 \mathrm{mmol})$, iodine (382 mg, 1.51 $\mathrm{mmol})$ and $\mathrm{NaH}(67 \mathrm{mg}, 2.86 \mathrm{mmol})$ as a colorless liquid (228 mg, $1.02 \mathrm{mmol}, 71 \%$ ) and a 1:2 cis/trans-mixture. trans-17e: ${ }^{1} \mathrm{H} \mathrm{NMR}\left(500 \mathrm{MHz}, \mathrm{CDCl}_{3}\right): \delta=3.25\left(\mathrm{dd},{ }^{3} J_{3,2}=7.3 \mathrm{~Hz},{ }^{3} J_{3,4}=1.9 \mathrm{~Hz}\right.$, $1 \mathrm{H}, 3-\mathrm{H}), 4.09\left(\mathrm{~d},{ }^{3} J_{4,3}=1.9 \mathrm{~Hz}, 1 \mathrm{H}, 4-\mathrm{H}\right), 5.41\left(\mathrm{~d},{ }^{3} J_{1(\text { trans }), 2}=10.4 \mathrm{~Hz}, 1-\mathrm{H}_{\text {trans }}\right), 5.59\left(\mathrm{~d},{ }^{3} J_{1(\text { cis }), 2}=\right.$ $\left.17.0 \mathrm{~Hz}, 1 \mathrm{H}, 1-\mathrm{H}_{\text {cis }}\right), 5.82\left(\mathrm{ddd},{ }^{3} J_{2,1(\text { cis })}=17.0 \mathrm{~Hz},{ }^{3} J_{2,1 \text { (trans })}=10.4 \mathrm{~Hz},{ }^{3} J_{2,3}=7.3 \mathrm{~Hz}, 1 \mathrm{H}, 2-\mathrm{H}\right), 7.17-$ 7.57 (m, 4H, 7-H, 8-H, 9-H, 10-H); ${ }^{13} \mathrm{C}$ NMR (125 MHz, $\left.\mathrm{CDCl}_{3}\right): \delta=59.9$ (d, C-4), $62.2(\mathrm{~d}, \mathrm{C}-3)$, 120.2 (t, C-1), 122.4 (s, C-6), 126.1 (d, C-8), 127.6 (d, C-10), 129.2 (d, C-9), 131.4 (s, C-5), 132.2 (d, C-7), 134.6 (d, C-2); cis-17e: ${ }^{1} \mathrm{H}$ NMR (500 MHz, $\left.\mathrm{CDCl}_{3}\right): \delta=3.18\left(\mathrm{dd},{ }^{3} J_{3,2}=5.6 \mathrm{~Hz},{ }^{3} J_{3,4}=4.1\right.$ $\mathrm{Hz}, 1 \mathrm{H}, 3-\mathrm{H}), 4.32\left(\mathrm{~d},{ }^{3} J_{4,3}=4.1 \mathrm{~Hz}, 1 \mathrm{H}, 4-\mathrm{H}\right), 5.26\left(\mathrm{~m}, 2 \mathrm{H}, 1-\mathrm{H}_{\text {trans }}, 2-\mathrm{H}\right), 5.57\left(\mathrm{~d},{ }^{3} J_{1(c i s), 2}=18.0\right.$ $\mathrm{Hz}, 1 \mathrm{H}, 1-\mathrm{H}_{\text {cis }}$ ), 7.17-7.57 (m, 4H, 7-H, 8-H, 9-H, 10-H); ${ }^{13} \mathrm{C} \mathrm{NMR}\left(125 \mathrm{MHz}, \mathrm{CDCl}_{3}\right): \delta=59.3(\mathrm{~d}$, C-4), 69.4 (d, C-3), 121.9 (t, C-1), 122.2 (s, C-6), 126.9 (d, C-8), 128.4 (d, C-10), 129.1 (d, C-9), 131.5 (s, C-5), 132.0 (d, C-7), 136.6 (d, C-2); HRMS (CI): calcd. for $\mathrm{C}_{10} \mathrm{H}_{10}{ }^{79} \mathrm{BrO}[\mathrm{M}+\mathrm{H}]^{+} 224.9915$; found 224.9896 .

trans-2-(4-Nitrophenyl)-3-vinyloxiran (17i): According to the general procedure for vinyl epoxide formation 17i was obtained from stannane $13 \mathbf{i}(270 \mathrm{mg}, 0.56 \mathrm{mmol})$, iodine (152 $\mathrm{mg}, 0.60 \mathrm{mmol})$ and $\mathrm{NaH}(27 \mathrm{mg}, 1.12 \mathrm{mmol})$ as pale yellow crystals $(56 \mathrm{mg}, 0.29 \mathrm{mmol}, 52 \%)$ and as a single stereoisomer; mp. $54-56{ }^{\circ} \mathrm{C}$, trans-17i: ${ }^{1} \mathrm{H}$ NMR (500 MHz, $\left.\mathrm{CDCl}_{3}\right): \delta=3.33\left(\mathrm{dd},{ }^{3} J_{3,2}=7.6 \mathrm{~Hz},{ }^{3} J_{3,4}\right.$ $=1.9 \mathrm{~Hz}, 1 \mathrm{H}, 3-\mathrm{H}), 3.85\left(\mathrm{~d},{ }^{3} J_{4,3}=1.9 \mathrm{~Hz}, 1 \mathrm{H}, 4-\mathrm{H}\right), 5.38\left(\mathrm{~d},{ }^{3} J_{1 \text { (trans }), 2}=10.4 \mathrm{~Hz}, 1 \mathrm{H}, 1-\mathrm{H}_{\text {trans }}\right), 5.55$ $\left(\mathrm{d},{ }^{3} J_{1(c i s), 2}=17.0 \mathrm{~Hz}, 1 \mathrm{H}, 1-\mathrm{H}_{\text {cis }}\right), 5.72\left(\mathrm{ddd},{ }^{3} J_{2,1(\text { cis })}=17.0 \mathrm{~Hz},{ }^{3} J_{2,1 \text { (trans })}=10.4 \mathrm{~Hz},{ }^{3} J_{2,3}=7.6 \mathrm{~Hz}\right.$, $1 \mathrm{H}, 2-\mathrm{H}), 7.44\left(\mathrm{~d},{ }^{3} J_{6,7}=8.8 \mathrm{~Hz}, 1 \mathrm{H}, 6-\mathrm{H}\right), 8.19\left(\mathrm{~d},{ }^{3} J_{7,6}=8.8 \mathrm{~Hz}, 1 \mathrm{H}, 7-\mathrm{H}\right) ;{ }^{13} \mathrm{C}$ NMR $(125 \mathrm{MHz}$, $\left.\mathrm{CDCl}_{3}\right): \delta=59.1(\mathrm{~d}, \mathrm{C}-4), 63.3(\mathrm{~d}, \mathrm{C}-3), 120.6$ (t, C-1), 123.8 (d, C-7), 126.2 (d, C-6), 134.1 (d, C-2), 144.5 (s, C-5), 147.8 (s, C-8); HRMS (CI): calcd. for $\mathrm{C}_{10} \mathrm{H}_{10} \mathrm{NO}_{3}[\mathrm{M}+\mathrm{H}]^{+}$192.0658; found 192.0659; $\mathrm{C}_{10} \mathrm{H}_{9} \mathrm{NO}_{3}$ (191.18): calcd. C 62.82, H 4.74, N 7.33; found C 62.97, H 4.91, N 7.15.

trans-2-Phenylmethyl-3-vinyloxiran (17k): According to the general procedure for vinyl epoxide formation 17k was obtained from stannane 13k $(385 \mathrm{mg}, 0.85 \mathrm{mmol})$, iodine (228 $\mathrm{mg}, 0.90 \mathrm{mmol})$ and $\mathrm{NaH}$ (48 mg, $2.00 \mathrm{mmol}$ ) as a colorless liquid (97 mg, $0.60 \mathrm{mmol}, 72 \%)$ and as a single stereoisomer; trans-17k: ${ }^{1} \mathrm{H}$ NMR (500 MHz, $\mathrm{CDCl}_{3}$ ): $\delta=2.80-2.84$ (m, 2H, 5-H, 5'-H), 2.99 (ddd, $\left.{ }^{3} J_{4,5}=5.7 \mathrm{~Hz},{ }^{3} J_{4,5}=5.3 \mathrm{~Hz},{ }^{3} J_{4,3}=1.9 \mathrm{~Hz}, 1 \mathrm{H}, 4-\mathrm{H}\right), 3.09\left(\mathrm{dd},{ }^{3} J_{3,2}=7.3 \mathrm{~Hz},{ }^{3} J_{3,4}=1.9 \mathrm{~Hz}, 1 \mathrm{H}, 3-\right.$ $\mathrm{H}), 5.17\left(\mathrm{dd},{ }^{3} J_{1(\text { trans }), 2}=9.8 \mathrm{~Hz},{ }^{2} J_{1(\text { trans }), 1(\text { cis })}=0.6 \mathrm{~Hz}, 1 \mathrm{H}, 1-\mathrm{H}_{\text {trans }}\right), 5.38\left(\mathrm{dd},{ }^{3} J_{1(\text { cis }), 2}=17.2 \mathrm{~Hz}\right.$, $\left.{ }^{2} J_{1(\text { cis }), 1(\text { trans })}=0.6 \mathrm{~Hz}, 1 \mathrm{H}, 1-\mathrm{H}_{\text {cis }}\right), 5.51\left(\mathrm{ddd},{ }^{3} J_{2,1(\text { cis })}=17.2 \mathrm{~Hz},{ }^{3} J_{2,1(\text { trans })}=9.8 \mathrm{~Hz},{ }^{3} J_{2,3}=7.3 \mathrm{~Hz}, 1 \mathrm{H}\right.$, 
2-H), 7.13-7.25 (m, 5H, 7-H, 8-H, 9-H); ${ }^{13} \mathrm{C}$ NMR (125 MHz, $\left.\mathrm{CDCl}_{3}\right): \delta=28.2(\mathrm{t}, \mathrm{C}-5), 58.5(\mathrm{~d}, \mathrm{C}-$ 4), 60.3 (d, C-3), 119.2 (t, C-1), 126.6 (d, C-7), 128.5 (d, C-9), 128.9 (d, C-8), 135.4 (d, C-2), 137.0 (s, C-6); HRMS (CI): calcd. for $\mathrm{C}_{11} \mathrm{H}_{13} \mathrm{O}[\mathrm{M}+\mathrm{H}]^{+}$161.0953; found 161.0960. 